\title{
A CHARACTERIZATION OF PRIME NOETHERIAN P.I. RINGS AND A THEOREM OF MORI-NAGATA
}

\begin{abstract}
AMIRAM BRAUN
ABSTRACT. Let $R$ be a noetherian prime p.i. ring, $C$ the center of $R$ and $\bar{C}$ its normalization. It is proved that $R$ is integral over its center iff $\bar{C}$ is a Krull domain. We also give a simple proof for the following theorem [7]: The normalization of a commutative noetherian domain is Krull.
\end{abstract}

Introduction. The purpose of this note is twofold. Firstly, to give an elementary simple proof of the so-called "Mori-Nagata" theorem [7]: "Let $C$ be a noetherian commutative domain, then $\bar{C}$-its normalization, is Krull." A generalization for the nonnoetherian case is also obtained. Secondly, we give a characterization for a noetherian prime P.I. (polynomial identity) ring $R$ to be integral over its center. One should note that there already exists a fairly simple proof for the Mori-Nagata theorem [8]. Unfortunately, this proof does not seem to be useful in the noncommutative case.

Our main theorem in $\S 2$ is that "A noetherian prime P.I. ring $R$ is integral over its center $C$, iff $\bar{C}$ is Krull." This result may be viewed as a generalization of the following theorem ([1], [2]): “A noetherian prime P.I. ring $R$ is finite over its center $C$, iff $C$ is noetherian." The special case of $C=\bar{C}$ is already obtained in [11].

The commutative results are handled in $\S 1$, while the noncommutative part is in $\S 2$. The characterization obtained in $\$ 2$ is a straightforward application of the methods in $\S 1$, hence we shall merely mention the difference between these proofs. All rings are with unit, and for notations we follow [5] in $\$ 1$ and [9] in $\$ 2$.

1. All rings are commutative. We shall give here a short elementary proof and a mild generalization to the following theorem.

TheOREM [7]. Let $C$ be a noetherian integral domain, then $\bar{C}$ is a Krull domain.

For a proof of the theorem the following result due to Matijevic is important.

Proposition 1.1 [6] $C$ as above, $K=\mathrm{qf}(C)$. Let $C^{\prime}=\{x \in K \mid \exists I$ an ideal in $C$ with $x I \subseteq C$ and $C / I$ is artinian $\}$. Then every subring $S, C \subset S \subset C^{\prime}$ is noetherian.

Received by the editors March 24, 1978.

AMS (MOS) subject classifications (1970). Primary 16A38; Secondary 13B20.

(c) 1979 American Mathematical Society 0002-9939/79/0000-0151/\$02.75 
REMARK 1.2. For a very short proof and a noncommutative generalization see [10].

Corollary 1.3 (KRUll-AKIZUKI [7]). Let $C$ be a noetherian domain, $\operatorname{dim}(C)=1$ then every overring $S, C \subset S \subset K=\mathrm{qf}(C)$ is noetherian.

We construct a sequence of rings $\left\{C_{\lambda}\right\}, \lambda$ an ordinal number, by means of transfinite induction. They will satisfy the following properties:

(i) $C \subset C_{\lambda} \subset \bar{C}$,

(ii) $\forall x$ in $C_{\lambda} \exists m_{1}=m_{1}(x), \ldots, m_{r}=m_{r}(x)$ with $x m_{1}^{e_{1}} \cdots m_{r}^{e_{r}} \subseteq C$, where $m_{i}, i=1, \ldots, r$, are maximal ideals of $C$ and $e=e_{1}(x), \ldots, e_{r}=$ $e_{r}(x)$ are in $\mathbf{N}$.

Say $C_{1}=C$. And assume $C_{\lambda}$ is defined. If for all maximal ideals $p$ in $C_{\lambda}$ either $\operatorname{grad}(p) \neq 1$, or $\operatorname{grad}(p)=1$ and $p \cdot p^{-1}=C_{\lambda}$ happens, we stop the process in the $\lambda$ step. Otherwise, we take $p$ maximal ideal in $C_{\lambda}$ with $\operatorname{grad}(p)=1$ and $p \cdot p^{-1}=p$ we define $C_{\lambda+1}=p^{-1}$. It is easy to check that $C_{\lambda+1}$ is a ring. If $\mu$ is a limit ordinal and $C_{\lambda}$ is defined $\forall \lambda<\mu$, we define $C_{\mu} \equiv \cup_{\lambda<\mu} C_{\lambda}$.

REMARK 1.4. (a) For $\rho<\nu$ we have $C_{\rho} \subsetneq C_{\nu}$.

(b) Each $C_{\rho}$ is noetherian.

Proof. (a) is easy.

(b) combines Proposition 1.1 and property (ii).

LEMMA 1.5. With the previous notation $C_{\mu}$ satisfies (i) and (ii).

Proof. Say $\mu=\lambda+1$, then $p \cdot p^{-1}=p$ and $C_{\lambda}$ is noetherian implies that $p^{-1} \subseteq \bar{C}$. For (ii) let $x \in C_{\lambda+1} . x p \subseteq p \subset C_{\lambda}$ and so $x(p \cap C) \subseteq C_{\lambda}$. Let $p \cap C=\left(a_{1}, \ldots, a_{s}\right)$. Then $x a_{i} \in C_{\lambda}$ for $i=1, \ldots, s$. Pick $v_{1}, \ldots, v_{l}$ maximal ideal in $C$ with $\left(x a_{i}\right) v_{1}^{t_{1}} \cdots v_{l}^{t_{t}} \subseteq C$ for $i=1, \ldots, s$ and then

$$
x(p \cap C) v_{1}^{t_{1}} \cdots v_{l}^{t_{l}} \subseteq C
$$

but $p \cap C$ is a maximal ideal in $C$ since $p$ is in $C_{\lambda}$.

Properties (i) and (ii) are easily checked for $\mu$-a limit ordinal. Q.E.D.

TheOREM 1.6. Let $C$ be a noetherian domain then $\bar{C}$ is Krull.

Proof. $C$ is noetherian implies that $C=\cap C_{q}$ where the intersection runs on all maximal grad one ideals $q$ and the intersection is locally finite [5]. Hence $\bar{C}=C^{*}=\cap C_{q}^{*}=\cap \bar{C}_{q}[4]\left(C^{*}\right.$-the complete integral closure of $\left.C\right)$, and obviously the intersection is still locally finite. Hence it suffices to show that each $\bar{C}_{q}$ is Krull; that is, we may assume that $K$-dim $C<\infty$ to begin with. We shall prove the theorem via induction on $\operatorname{Krull}-\operatorname{dim}(C)$. The case $K$-dim $C=1$ is established in Corollary 1.3. We apply now the process of constructing $\left\{C_{\lambda}\right\}$ from $C$. If the process does not stop we exhaut $\bar{C}$ after a step $\mu$ with $\operatorname{card}(\mu)>\operatorname{card}(\bar{C})$, and so $\bar{C}=C_{\mu}$ is noetherian, hence Krull. Say the process stops at $\mu$; then $\forall p$ a maximal ideal in $C_{\mu}$ either $\operatorname{grad}(p) \neq 1$ or if $\operatorname{grad}(p)=1, p \cdot p^{-1}=C_{\mu}$ and then $h(p)=1$. Now, $C_{\mu}$ is noetherian; 
hence $C_{\mu}=\cap_{q}\left(C_{\mu}\right)_{q}$ where $\operatorname{grad}(q)=1$ and the intersection is locally finite. But

$$
\operatorname{dim}\left(C_{\mu}\right)_{q}<\operatorname{dim} C_{\mu}=\operatorname{dim} C
$$

for each such $q$ hence by induction $\overline{\left(C_{\mu}\right)_{q}}$ is Krull. Again,

$$
\bar{C}=\bar{C}_{\mu}=C_{\mu}^{*}=\bigcap\left(C_{\mu}\right)_{q}^{*}=\bigcap \overline{\left(C_{\mu}\right)_{q}}
$$

and the intersection is locally finite, hence $\bar{C}$ is Krull. Q.E.D.

The next lemma, definitely well known, is left as an exercise to the reader.

Lemma 1.7. Let $C$ be a domain, $S \subseteq C$ a multiplicative closed set, and $p_{S}$ is a prime ideal in $C_{S}$ with $\operatorname{grad}\left(p_{S}\right)=1$, then $\operatorname{grad}(p)=1$.

THEOREM 1.8. Let $C$ be a domain such that $C_{p}$ is noetherian for all prime ideals $p$ in $C$, then

(1) $\bar{C}=\cap \bar{C}_{\bar{p}}$, the intersection is on all prime ideals $\bar{p}$ in $\bar{C}$ with $h(\underline{p})=1$;

(2) $\bar{C}_{\bar{p}}$ is a D.V.R. for all $\bar{p}, h(\bar{p})=1$ where $\bar{p}$ is a prime ideal in $\bar{C}$.

Proof. We observe first that $h(\bar{p})<\infty$ for all prime ideals $\bar{p}$ in $\bar{C}$. Indeed let $p=\bar{p} \cap C$ then $K$-dim $C_{p}<\infty$, and so $h(\bar{p}) \leqslant K$-dim $\bar{C}_{p}<\infty$. We prove (1) by induction on $h(\bar{p})$. More precisely, we prove inductively that for $\bar{p}$ prime ideal in $\bar{C}$ and $\operatorname{grad}(\bar{p})=1, \bar{C}_{\bar{p}}=\cap \bar{C}_{\bar{v}}$, where $\bar{v}$ are prime ideals in $\bar{C}$ and $h(\bar{v})=1$. Given $\bar{p}$ in $\bar{C}, \operatorname{grad}(\bar{p})=1$. Let $S=\bar{C} \backslash \bar{p}, S_{1}=C \backslash p$. If $\operatorname{grad}\left(\bar{p}_{S}\right)=1$ by Lemma $1.7, \operatorname{grad}\left(\bar{p}_{S_{1}}\right)=1$, but

$$
\bar{p}_{S_{1}} \triangleleft(\bar{C})_{S_{1}}=\left(\overline{C_{S_{1}}}\right) \equiv \overline{C_{p}}
$$

is Krull (Theorem 1.6) hence $h\left(\bar{p}_{S_{1}}\right)=1$ and consequently $h(\bar{p})=1$. We may therefore assume that $\operatorname{grad}\left(\bar{p}_{S}\right)>1$ and so $\bar{C}_{\bar{p}} \equiv \bar{C}_{S}=\bigcap\left(\bar{C}_{S}\right)_{W}, W \triangleleft \overline{C_{S}}$ and $\operatorname{grad}(W)=1$. Clearly $W=V_{S}$ where $V$ is an ideal in $\bar{C}$ and $V \subsetneq \bar{p}$. Now $\operatorname{grad}(W)=1=\operatorname{grad}\left(V_{S}\right)$ and we apply again Lemma 1.7 to get that $\operatorname{grad}(V)$ $=1$. We can apply our induction hypothesis on $V(h(V)<h(\bar{p}))$ and so $\bar{C}_{V} \equiv \cap \bar{C}_{\bar{q}}, h(\bar{q})=1, \bar{q}$ are prime ideals in $\bar{C}$. All in all $\left(\bar{C}_{\bar{p}}\right)_{W}=\bar{C}_{V}=$ $\cap \bar{C}_{\bar{q}}, h(\bar{q})=1$, and so $\bar{C}_{\bar{p}}=\bar{C}_{S}=\cap \bar{C}_{\bar{q}}, h(\bar{q})=1, \bar{q}$ is a prime ideal of $\bar{C}$. To prove (2) we let $\bar{p} \subseteq \bar{C}$ be a prime ideal in $\bar{C}$ and, $h(\bar{p})=1$ and $p=\bar{p} \cap C, S_{1}=C \backslash p$. Then $\bar{p}_{S_{\perp}}$ is a prime ideal of $\overline{C_{S_{1}}}$ and $h\left((\bar{p})_{S_{1}}\right)=1$, and $\bar{C}_{S_{1}}$ being Krull implies that $\left(\bar{C}_{S_{1}}\right)_{\bar{p}_{S_{1}}}=\bar{C}_{\bar{p}}$ is a D.V.R. Q.E.D.

The next theorem is a direct consequence of the preceding one.

THEOREM 1.9. Let $C$ be a domain satisfying the following conditions:

(1) $C_{p}$ is noetherian for every prime ideal $p$ in $C$.

(2) $C=\cap_{q} C_{q}$, where $q$ is a maximal grade one prime ideal and the intersection is locally finite.

Then $\bar{C}$ is Krull. 
Proof. That $C=\cap_{q} C_{q}$ and the intersection is locally finite implies that $C^{*}=\cap C_{q}^{*}[4]$, moreover, $C_{q}$ is noetherian; hence, $C_{q}^{*}=\bar{C}_{q}=\cap \bar{C}_{\bar{p}}$ where $h(\bar{p})=1, \bar{p}$ is a prime ideal in $\bar{C}$ and $\bar{p} \cap C \subseteq q$ for some $q$. We claim that every prime $\bar{p}$ in $\bar{C}$, with $h(\bar{p})=1$ appears in this intersection. Indeed, let $\bar{p}$ be such ideal and $p \equiv \bar{p} \cap C$ then $\operatorname{grad}\left(\bar{p}_{p} \cap C_{p}\right)=1$ [7]; hence $\operatorname{grad}\left(p_{p}\right)=1$ and so $\operatorname{grad}(p)=1(1.7)$, but then $p \subset q, q$ a maximal ideal of grade one. Thus $\bar{p}_{q}$ is a prime ideal in $\bar{C}_{q}$ and $h\left(\bar{p}_{q}\right)=1$ and so $\left(\bar{C}_{q}\right)_{\bar{p}_{q}}=\bar{C}_{\bar{p}}$ appears in the intersection $\cap\left(\bar{C}_{q}\right)_{W}, h(\bar{W})=1, \bar{W}$ is prime in $\bar{C}_{q}$. Condition (1) and 1.8 guarantees that $\bar{C}=\cap \bar{C}_{\bar{p}}, h(\bar{p})=1$ and $\bar{p}$ is prime in $\bar{C}$, hence $\bar{C}=C^{*}$; that is, $\bar{C}$ is Krull.

2. We switch now to the noncommutative analog of $\S 1$ in order to prove the following:

THEOREM 2.1. Let $R$ be a noetherian prime P.I. ring, then $R$ is integral over its center $C$ iff $\bar{C}$ is a Krull domain.

The proof will follow closely the pattern of the previous section and so we shall merely emphasize the places where an additional argument is required. We begin by stating three lemmas, the proofs of which are given later.

Lemma 2.2. Let $R$ be as in Theorem 2.1, then $C=\cap_{p} C_{p}$ where the intersection runs on all maximal grade one prime ideals $p$, and the intersection is locally finite.

Lemma 2.3 [11]. $R$ as in Theorem 2.1. Let $x \in K$-the quotient field of $C$ and $x M \subseteq M$ where $M=m_{1} R+\cdots+m_{k} R$ a right $R$ module and $m_{i} r=r m_{i}$ for each $r$ in $R$, then $x$ is integral over $C$.

Lemma 2.4. $R$ as in Theorem 2.1, $p$ a prime ideal in $C$ then $h(p)<\infty$.

Corollary 2.5. Let $R$ be as in Theorem 2.1 , then $\bar{C}=C^{*}$-the complete integral closure of $C$.

Proof. Let $x \in C^{*}$ and $a \in C$ with $a x^{n} \in C$ for $n=1,2, \ldots$ Then $x^{n} \in R 1 / a$ for $n=1,2, \ldots$, and so $R \subset R[x] \subset R 1 / a$. $R$ being noetherian implies that $R[x]=R v_{1}+\cdots+R v_{s}$ and we may take $v_{i}$ to be a polynomial in $x$, hence $v_{i} \in Z(R[x])$. We apply Lemma 2.3 with $M=R[x]$ and get that $x \in \bar{C}$. The other inclusion is trivial.

ProOF OF THEOREM 2.1. We define via transfinite induction the sequence $\left\{R_{\lambda}\right\}$, obeying the following conditions:

(1) $R \subseteq R_{\lambda} \subset R \bar{C}$.

(2) For $x$ in $R_{\lambda}, \exists m_{1}, \ldots, m_{r}$ maximal ideals in $C$, and $i_{1}, \ldots, i_{r}$, such that $x m_{1}^{i_{1}} \cdots m_{r}^{i_{r}} \subseteq R$.

(3) $R_{\lambda}$ is integral over $C_{\lambda}$ and $C_{\lambda} \subset \bar{C}$ where $C_{\lambda}=Z\left(R_{\lambda}\right)$. We observe first that once $R_{\lambda}$ is defined then $R_{\lambda}$ is left and right noetherian [10]. Given an ordinal $\mu$ and assume that $R_{\lambda}$ is defined for each $\lambda<\mu$ we define $R_{\mu}$. 
Case (i). $\mu=\lambda+1$ then $R_{\mu} \equiv m_{0}^{-1} R_{\lambda}$ where $m_{0}$ is a maximal ideal in $C_{\lambda}$, $\operatorname{grad}\left(m_{0}\right)=1$ and $m_{0}^{-1} \cdot m_{0}=m_{0}$. The absence of such maximal $m_{0}$ will stop the process and we would not define $R_{\mu}^{\sim}$.

Case (ii). $\mu=\mathrm{a}$ limit ordinal then $R_{\mu}={ }_{\lambda<\mu} R_{\lambda}$. It is easy to check that $R_{\mu}$ is a ring and that (1) is satisfied. To check (2) we see first that for $\mu$ a limit ordinal it is trivial. If $\mu=\lambda+1$ we pick $a_{1}, \ldots, a_{r}$ in $C$ such that $m R=a_{1} R$ $+\cdots+a_{r} R$ where $m=m_{0} \cap C$ and then $x a_{i} \in R_{\lambda}$ for $i=1, \ldots, r$, and there are $m_{1}, \ldots, m_{t}$ such that $\left(x a_{i}\right) \Pi_{j=1}^{t} m_{j}^{i_{j}} \subseteq R$ and so $x m \Pi_{j=1}^{t} m_{j}^{e_{j}} \subseteq R, m$ is maximal in $C$ since $C_{\lambda} \subseteq \bar{C}$.

To check (3) we see again that $\mu=a$ limit ordinal is easy. Indeed, let $x \in R_{\mu}$ then $x \in R_{\lambda}$ for $\lambda<\mu, x$ is integral over $C_{\lambda} \subseteq C_{\mu}$. Say $\mu=\lambda+1$ then $R_{\lambda+1}=m_{0}^{-1} R_{\lambda} \subseteq R_{\lambda} 1 / y$ where $y \in m_{0}$ for some $y$, then $R_{\lambda}$ being noetherian implies

$$
R_{\lambda+1}=R_{\lambda} w_{1}+\cdots+R_{\lambda} w_{l}
$$

and we can take $w_{i} \in m_{0}^{-1} \subseteq Z\left(R_{\lambda+1}\right)$. Let $x \in C_{\lambda+1}$ then by Lemma $2.3 x$ is integral over $C_{\lambda}$ and in particular $w_{i}, i=1, \ldots, l$, are so. Let $y \in R_{\lambda+1}$ then $y=\sum t_{i} w_{i}, i=1, \ldots, l$. But $t_{1}, \ldots, t_{l} \in R$ are integral over $C_{\lambda}$ and we have that

$$
D=C_{\lambda}\left[t_{1}, \ldots, t_{l}, w_{1}, \ldots, w_{l}\right]
$$

is a finite $C_{\lambda}$ module [9], now $y \in D$ and so $y$ is integral over $C_{\lambda}$ by the determinant argument. This also establishes the inclusion $C_{\lambda} \subset C_{\lambda+1} \subset \bar{C}$.

The proof follows now the same lines as in $\$ 1$ via induction on $\operatorname{dim} R=$ $\operatorname{dim} C<\infty$, the reduction to this case is by Lemmas 2.2 and 2.4, the case $\operatorname{dim} R=1$ is valid by Remark 1.2. We need two additional remarks. First, if the $R_{\lambda}$ sequence does not stop, then $R_{\lambda}=R \bar{C}$ for some $\mu$ and $R_{\mu}$ is noetherian and integral over its center $C_{\mu} \subset \bar{C}$; but then $\bar{C}=C_{\mu}$ and $R \bar{C}$ is centrally integrally closed in the sense of [11] and hence $\bar{C}$ is Krull [11].

Secondly, the following remark is needed: Let $q \triangleleft C_{\lambda}$ with $\operatorname{grad}(q)=1, q$ is a maximal ideal and $q^{-1} \cdot q=C_{\lambda}$ then $h(q)=1$. Indeed, we may localize by $q$, if $p \subset q$, prime ideal in $C_{\lambda}$, then $\left(q^{-1} \cdot p\right) q \subset p$, hence $q^{-1} \cdot p \subset p$, hence $q^{-1} \cdot p=p$ or $p=q p$, hence $p R_{\lambda}=q\left(p R_{\lambda}\right)$. But $q \subset J\left(R_{\lambda}\right)$ since $q$ is maximal and $R_{\lambda}$ is integral over $C_{\lambda}$ and so we get a contradiction via Nakayama's Lemma. Q.E.D.

We have to prove the converse: Let $R$ be a noetherian prime P.I. ring and $\bar{C}$ is Krull, then $R$ is integral over $C$. From [11] we have that $C^{*} \supset Z(T(R))$ where $T(R)$ is the trace-envelope of $R$ and so $(\bar{C})^{*} \supset Z(T(R))$ but $(\bar{C})^{*}=\bar{C}$ $\left(\bar{C}\right.$ being Krull) implies that $\bar{C} \supset Z(T(R))$. Thus for $r \in R, c_{i}(r), i=$ $1, \ldots, n$, the coefficients of its characteristic polynomial are integral over $C$ and so $r$ is integral over $C$. Q.E.D.

Note 2.6. One can get a better criterion by merely requiring that $\bar{C}=$ $\cap_{\bar{v}} \bar{C}_{\bar{v}}, h(\bar{v})=1$ and $\bar{C}_{\bar{v}}$ is a D.V.R. The proof, though, is more complicated. 
Moreover, if $\bar{C}$ is completely integrally closed, no noetherian condition is needed to show that $R$ is integral over $C$ [11].

REMARK 2.7. There is an example [11] of a noetherian prime P.I. ring, integral over its center and is not a finite module over it.

We need to prove Lemmas 2.2, 2.3, and 2.4.

Proof of Lemma 2.2. That $C=\bigcap_{p} C_{p}$ where $\operatorname{grad}(p)=1$ is always true [5]. We prove that the intersection is locally finite. We follow Kaplansky's commutative proof [5]. Firstly, if $p$ is maximal with respect to the principal ideal $(x)$ then there exists a prime ideal $P$ in $R$ and $y \in C$ such that $P \supseteq\{r \in R \mid y r \in x R\}$ and $y \notin x C$ and so $p=\{z \in C \mid z y \in x C\}$ where $p=P \cap C[11]$. We show that every regular sequence in $p$ is of length one. $\{x\}$ is obviously a maximal sequence. Let $z \in p$ be another element, we shall show that $p \subset Z(C / z C)$. We have $p y \subseteq x C, y \notin x C$ implies $z \cdot y \in x C$ or $z y=x v$. We claim that $v \notin z C$ and $p v \subseteq z C$. Indeed if $v=z w, z y=x z w$ or $y=x w$ a contradiction. Also, $x p v=(x v) p=z y p \subseteq z x c$; hence, $p v \subseteq z C$. To finish the proof, let $x \in p, \operatorname{grad}(p)=1$ and $p$ is maximal above $y$. Then by the previous argument we have that $p \subset Z(C / x C)$. If $\exists p_{1} \supsetneq p$ prime and maximal via $p_{1} \subset Z(C / x C)$ then $p_{1} \subset Z(C / y C)$ contradicting the maximality of $p$, hence $p$ is maximal of grade one over $x$. There are only finitely many such ideals [11], hence $x$ belongs to only finitely many. Q.E.D.

Proof of LemMa 2.3. This is an immediate corollary of [11].

Proof of LemMa 2.4. First we prove the following:

LEMMA (a). Let $R$ be a noetherian prime P.I. ring, integral over a central subring $D$. Then $D$ satisfies the basic Principal Ideal Theorem.

Proof. Let $p$ be a prime ideal in $D$ minimal above $x \in p$ and let $P$ be a prime ideal in $R$ with $P \cap R=p$ (there are such since $R$ is integral over $D$ ), then $P$ is minimal over $x(R$ is integral over $D)$ and so $h(P)=1$ [3]. This implies that $\operatorname{dim} C_{p}=\operatorname{dim} R_{p}=1$. Q.E.D.

We proceed now to prove the generalized Principal Ideal Theorem [5] for $D$. The proof is almost identical with the one in [5].

LemMa (b). Let $R$ be as in Lemma (a), then $C=Z(R)$ satisfies the generalized Principal Ideal Theorem.

Proof. Let $p$ be a prime ideal in $C$ minimal above $\left(a_{1}, \ldots, a_{n}\right)$ we prove via induction on $n$ that $h(p) \leqslant n$. The case $n=1$ is provided by Lemma (a). We localize $C$ at $p$ and assume that $C$ is local with $p$ the maximal ideal. Say $h(p)>n$ and let $p=p_{0} \supsetneqq p_{1} \supsetneqq p_{2} \supsetneqq \cdots \supsetneqq p_{n+1}$ a proper chain of length $n+1$ and no primes between $p$ and $p_{1}$ (we can do it since $R$ is integral over $C$ and noetherian). Obviously $\left(a_{1}, \ldots, a_{n}\right) \not \subset p_{1}$ say $a_{1} \notin p_{1}$ then $p$ is the only minimal prime above $\left(a_{1}, p_{1}\right)$ and therefore $p_{1} /\left(a_{1}, p_{1}\right)$ is a nil ideal in $C /\left(a, p_{1}\right)$, and we can find $t$ such that

$$
a_{i}^{t}=c_{i} a_{1}+b_{i}, \quad i=2, \ldots, n,
$$


and $b_{i} \in p_{1}, c_{i} \in C$. Let $J=\left(b_{2}, \ldots, b_{n}\right) \subset p_{1}$. We find by induction $q \subset p_{1}$ with $q \supset J$ and $h(q) \leqslant n-1$. Let $Q$ be a prime ideal of $R$ with $Q \cap C \neq q$. Then $R / Q$ is integral over $C / q$ but $p / q$ a minimal prime over $\left(a_{1}+q\right) / q$ is not of height one, a contradiction to Lemma (a).

To complete the proof of Lemma 2.4 one should observe that for $p$ a prime ideal in $C, p R=a_{1} R+\cdots+a_{l} R$ and that $p$ is minimal above $\left(a_{1}, \ldots, a_{l}\right)$.

\section{REFERENCES}

1. D. Eisenbud, Subrings of Artinian and Noetherian rings, Math. Ann. 185 (1970), 247-249.

2. E. Formanek, Noetherian P. I. rings, Comm. Algebra 1 (1974), 79-86.

3. A. V. Jategaonkar, Principal ideal theorem for Noetherian P.I. rings, J. Algebra 35 (1975), 17-22.

4. W. Heinzer, J. Ohm and R. L. Pendleton, On integral domains of the form $\cap D_{p}, p$ minimal, J. Reine Angew. Math. 241 (1970), 147-159.

5. I. Kaplansky, Commutative rings, Allyn and Bacon, Boston, Mass., 1970.

6. Jacob R. Matijevic, Maximal ideal transform of Noetherian rings, Proc. Amer. Math. Soc. 54 (1976), 49-52.

7. M. Nagata, Local rings, Interscience, New York, 1962.

8. J. Nishimura, Note on integral closures of a Noetherian integral domain, J. Math. Kyoto Univ. 16 (1976), 117-122.

9. C. Procesi, Rings with polynomial identity, Dekker, New York, 1973.

10. W. Schelter, On the Krull-Akizuki theorem, J. London Math. Soc. 13 (1976), 263-264.

11. __ Integral extensions of rings satisfying a polynomial identity, J. Algebra 40 (1976), 245-257.

Department of Mathematics, Rutgers University, New Brunswick, New Jersey 08903 